\title{
Film Casting of Viscoelastic Liquid
}

\author{
SEYED M. ALAIE and T. C. PAPANASTASIOU* \\ Department of Chemical Engineering \\ The University of Michigan \\ Ann Arbor, Michigan 48109
}

\begin{abstract}
Melt film-casting is analyzed by means of a nonlinear integral constitutive equation that incorporates shear history effects, spectrum of relaxation times, shear thinning, and extension thinning or thickening. Film temperature and thickness profiles are predicted. The effects of shear-thinning, relaxation time, and die-design on the final film thickness are evaluated. Temperature variations are investigated by means of the pseudo-time concept. The process dependence on the rheological characteristics of the viscoelastic fluid, the die-design through the history effects, the conditions at the take-up end, and the film temperature is discussed. Predicted film and temperature profiles of casting of polypropylene are compared with experimental data taken from the literature.
\end{abstract}

\section{INTRODUCTION}

$\mathbf{T}$ he film casting process is an important industrial operation to manufacture polymeric films and metal sheets (1). The process can be carried out with a polymer melt under its decomposition temperature, or else with a polymer solution. The polymer melt is extruded through center-fed "T" or coat hanger dies under pressure. Design equations for these dies were given by Clarke (2) for Newtonian liquid and by Pearson (1) for power law liquids. Upon exiting the die, the sheet initially swells and then its thickness decreases monotonically. The resulting flat film is usually stretched and oriented uniaxially while being cooled by convected cold air or inert gas and subsequent passage over chilled rolls.

Unlike the corresponding axisymmetric situation, the common fiber-spinning process, experimental, and theoretical studies on film casting are limited. Example of experimental investigation of steadycasting includes that by Kase, et al. (3). Theoretical investigations include the derivation of the approximate one-dimensional equations by Pearson (1) and stability analyses around the observed phenomenon of draw resonance by Kase (3) and Anturkar, et al. (4). More elaborate analyses of curtains of Newtonian liquids under gravity, surface tension, inertia, and applied tension were advanced by Kistler and Scriven (5) and by Adachi (6).

Here, casting of polymer melts under isothermal and non-isothermal conditions is considered. The process is analyzed by means of a BKZ-type integral constitutive equation, designed by Papanastasiou, et al. (7), which accounts for multiple relaxation times, shear-thinning, extension-thinning or thickening and for the shear-prehistory inside the die. In

\footnotetext{
- To whom correspondence should be addressed.
}

order to investigate the effects of temperature on the process, both the energy and momentum equations are solved, so that both velocity (and thickness) and temperature profiles are calculated simultaneously. The change of rheological properties of the polymer, due to temperature variation is modeled by means of "the shifted time scale" (8). Although a particular integral model is used here, the analysis that follows applies directly to any other integral constitutive equation of the BKZ (9) and Wagner (10) type that have been put forward, as well as to those developed from molecular theory $(11,12)$.

\section{GOVERNING EQUATIONS}

A planar film of viscoelastic liquid is extruded through a die of thickness $2 h_{0}$, and is continuously taken-up at length $L$ downstream, by drawing force $F$, at a velocity, $u_{L}=w R$, which is greater than the extrusion velocity, while being cooled by air as shown in Fig. 1. Extrudate swelling just downstream the die may be neglected for a thin and long film so that the governing equations can be averaged over the crosssection of the film to derive one-dimensional, approximate equations, as follows.

The steady-state, continuity equation is

$$
2 \rho w h u=\rho Q=\text { const. },
$$

where $Q$ is the volumetric flow rate, $u$ the mean velocity in the drawing direction $z, 2 h$ the film thickness, $w$ the film width, and the density $\rho$ is given by (13),

$$
\rho=\frac{\rho_{o}}{1+c_{\rho}\left(T-T_{0}\right)},
$$

where $\rho_{o}$ is the density at temperature $\mathrm{T}_{0}$ and $c_{\rho}$ a constant of expansion. For highly viscous polymer, 


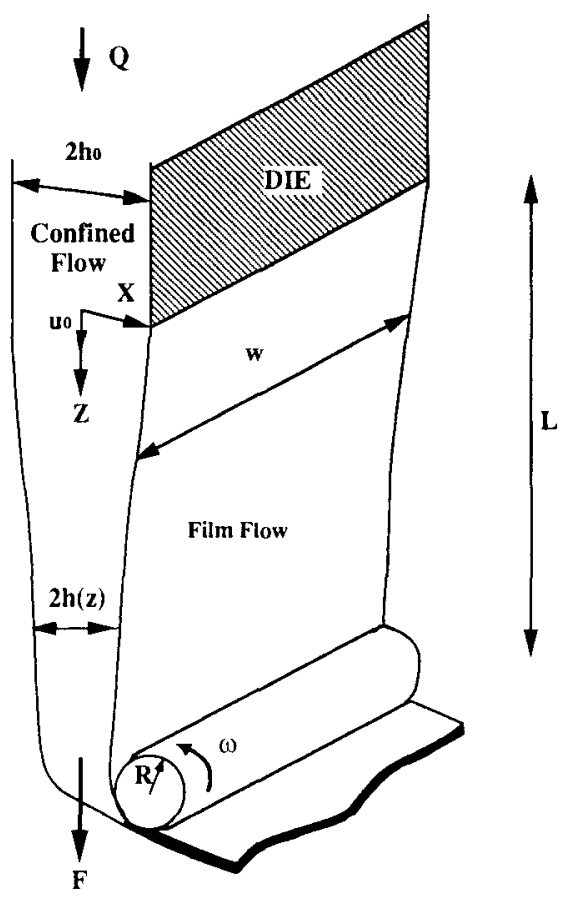

Fig. 1. Schematic of the process of film casting. A viscoelastic liquid is extruded through a die and is taken up at position $L$ downstream by a drawing force $F$ at a higher velocity $u_{L}\left(u_{L}>u_{0}\right) . u O$ is the velocity of the film at the inlet, or die-exit.

viscous effects dominate the process so that inertia, gravity, and surface tension effects can be neglected and the momentum equation takes the form

$$
\frac{d}{d z}\left[\left(\tau_{z z}-\tau_{x x}\right) h\right] w=0,
$$

where $\tau_{z z}$ and $\tau_{x x}$ are the normal stresses in the $z$ and the $x$ directions, respectively. Equation 3 simply states that the applied tension is transmitted unchanged along the film. By assuming uniform temperature $T$ over the cross section of the film and negligible axial heat conduction, viscous dissipation, and radiation, the energy equation simplifies to

$$
u \frac{d T}{d z}=-\frac{H}{c_{p} h}\left(T-T_{\mathrm{arr}}\right)
$$

where $T_{\text {air }}$ is the air temperature, $c_{p}$ the specific heat capacity, and $H$ the heat transfer coefficient. In order to account for the temperature dependency, the following expression, suggested by Haw (14), was used

$$
c_{p}(T)=\frac{c_{p}^{o} k_{1}+k_{2} T}{k_{1}+k_{2} T_{0}},
$$

where $c_{p}^{o}$ is the heat capacity at a reference temperature $T_{0}$, and $k_{1}$ and $k_{2}$ empirical constants. The chosen values for the constants in Eqs 2 and 5 are recorded in Table 1 . The heat transfer coefficient, $H$, was calculated by means of the relations (15).

$$
N u_{L}=a R a_{L}{ }^{1 / 3}, \quad R a \geq 10^{9}
$$

Table 1. Thermophysical Properties from (14).

\begin{tabular}{lll}
\hline$c \rho$ & 0.00069 \\
$k_{1}$ & 1.45 \\
$k_{2}$ & $0.00271\left(K^{-1}\right)$ \\
\hline
\end{tabular}

or

$$
N u_{L}=b R a_{L}^{1 / 4}, \quad R a \geq 10^{9}
$$

where, $R a_{L}=g \beta \Delta T L^{3} / v \gamma$, is the Rayleigh number and, $N u_{L}=H L / K$, the Nusselt number with $\alpha=K / \rho C_{p}$ the thermal diffusivity and $v=\mu / \rho$ the kinematic viscosity of the cooling air. Also, $\Delta T=T-T_{\text {alr }}$, is the driving temperature difference and, $T_{f}=1 / 2\left(T+T_{\text {air }}\right)$, the temperature where air-properties are estimated. The required boundary conditions are: At the inlet, at $z=0$, the velocity, $u=u_{0}$, and the temperature, $T=T_{0}$, are specified. At the outlet, at $z=L$, the draw velocity, $u=u_{L}$, or else the drawing force, $F=$ $\left(\tau_{z z}-\tau_{x x}\right) h w$, are specified.

\section{CONSTITUTIVE EQUATION}

To proceed with the analysis the stresses, $\tau_{z z}-\tau_{x x}$. in $E q 3$ must be expressed in terms of the velocity by means of a constitutive equation. A constitutive equation describing accurately the behavior of viscoelastic liquid is the primary element in the analysis of any viscoelastic flow. The rheology of a viscoelastic liquid is well approximated by integral constitutive equations which express the stress tensor explicitly in terms of local and upstream deformations and kinematics along streamlines. The stress difference in Eq 3 is expressed here by means of the PSM model (7) of the form

$$
\begin{aligned}
\tau=\int_{-\infty}^{t} \sum_{k=1}^{k} \frac{a_{k}}{\lambda_{k}} \exp \left(-\frac{t-t^{\prime}}{\lambda_{k}}\right) \\
\cdot \frac{\alpha}{(\alpha-3)+\beta \mathrm{I}_{B}+(1-\beta) \mathrm{II}_{B}}\left(B_{t}\left(t^{\prime}\right)-\mathrm{I}\right) d t^{\prime},
\end{aligned}
$$

where $\left\{a_{k}, \lambda_{k}\right\}$ is the relaxation spectrum, $\alpha$ and $\beta$ are material parameters, and $B$ the finger tensor. In isothermal casting the transit times are given in terms of the velocity and the distance by

$$
t-t^{\prime}=\int_{z^{\prime}}^{z} \frac{d z^{\prime \prime}}{u\left(z^{\prime \prime}\right)}
$$

and

$$
d t=\frac{d z}{u(z)},
$$

which transform the constitutive equation to its spatial form,

$$
\begin{aligned}
\tau= & \int_{-\infty}^{z} \sum_{k=1}^{k} \frac{a_{k}}{\lambda_{k}} \exp \left[\frac{-\int_{z^{\prime}}^{z} d z^{\prime \prime} /\left(u\left(z^{\prime \prime}\right)\right)}{\lambda_{k}}\right] \\
& \cdot \frac{\alpha}{(\alpha-3)+\beta \mathrm{I}_{B}+(1-\beta) \mathrm{I}_{B}}\left(B_{Z}\left(z^{\prime}\right)-\mathrm{I}\right) \frac{d z^{\prime}}{u\left(z^{\prime}\right)} .
\end{aligned}
$$

Equation 11 yields the local stress at distance, $z$, in 
terms of the deformation at earlier positions $z^{\prime}<z$ along the film (streamline). The stress on a fluid particle, $\tau(z)$, which at present time, $t$, occupies the position, $z$, is a function of the relative deformation tensor, $B_{\mathrm{z}}\left(z^{\prime}\right)$, to which the particle had been exposed in moving from far away to its present position $z$ along the streamline $-\infty<z^{\prime}<z$, as depicted in Fig. 2. The integral constitutive equation involves an unbounded domain of integration, from $-\infty$ to $z$, which we break into two subdomains as shown in Fig. 2. In the first subdomain, which represents contributions to local stress from the shear prehistory, the kinematics are those of a shear-thinning liquid, described by the same constitutive equation in channel flow, which yields velocity

$$
v=c\left[1-\left(\frac{x}{h_{0}}\right)^{n+1 / n}\right]
$$

where $c$ depends on the pressure gradient, $n$ is the degree of shear-thinning, and $h_{0}$ the die-half thickness. By demanding continuity of velocity at the exit of the die, it permits the evaluation of the parameter $c$, as

$$
c=\frac{h_{0}^{(n+1) / n}}{m((n+1) / n)}\left(\rho g-\frac{d p}{d z}\right),
$$

where $\rho$ is the density, $h_{0}$ the die-half width, and $m$ the coefficient of the power law equation. The constitutive equation then takes the form

$$
\begin{aligned}
\tau= & \sum_{k=1}^{k} \frac{a_{k}}{\lambda_{k}} \int_{-\infty}^{o} \exp \left[-\frac{\int_{z^{\prime}}^{o}\left(d z^{\prime \prime} / v\right)+\int_{0}^{z}\left(d z^{\prime \prime} / u\left(z^{\prime \prime}\right)\right.}{\lambda_{k}}\right] \\
& \cdot H\left(I_{B}, I I_{B}\right)\left(B_{[v, u(z)]}-I\right) \frac{d z^{\prime}}{v} \\
& +\sum_{k=1}^{k} \frac{a_{k}}{\lambda_{k}} \int_{0}^{z} \exp \left[-\frac{\int_{z^{\prime}}^{z}\left(d z^{\prime \prime} / u\left(z^{\prime \prime}\right)\right.}{\lambda_{k}}\right] \\
& \cdot H\left(I_{B}, I I_{B}\right)\left(B_{\left[u\left(z^{\prime}\right), u(z) \mid\right]}-I\right) \frac{d z^{\prime}}{u\left(z^{\prime}\right)} .
\end{aligned}
$$

To relate the viscoelastic stress difference, $\tau_{z z}-\tau_{x x}$, to the three involved forms of the velocity $\left[u(z), u\left(z^{\prime}\right)\right.$, $v]$, the Finger tensors $B\left[u\left(z^{\prime}\right), u(z)\right]$ and $B[u(z), v]$ must be expressed in terms of these velocities. To do so we solve the relative deformation equation in one dimension, by following a procedure similar to the one outlined elsewhere $(18,19)$. The resulting Finger tensors needed in the two integrals of $E q 18$ are:

(a) $B[v, u(z)]$, between the present location $z$ of velocity $u(z)$ (within the extensional field $0 \leq z \leq L$ ) and past states in the shear prehistory of $-\infty \leq z^{\prime} \leq 0$ of velocity $v$ :

$B(v, u(z))$

$$
=\left[\begin{array}{ccc}
{\left[\frac{u(0)}{u(z)}\right]^{2}} & 0 & {\left[\frac{d v}{d x} \frac{\left(0-z^{\prime}\right)}{v}\right]} \\
0 & 1 & 0 \\
{\left[\frac{d v}{d x} \frac{\left(0-z^{\prime}\right)}{v}\right]} & 0 & \left(\frac{u(z)}{u(0)}\right)^{2}\left[1+\left(\frac{d v}{d x} \frac{\left(0-z^{\prime}\right)}{v}\right)^{2}\right]
\end{array}\right]
$$

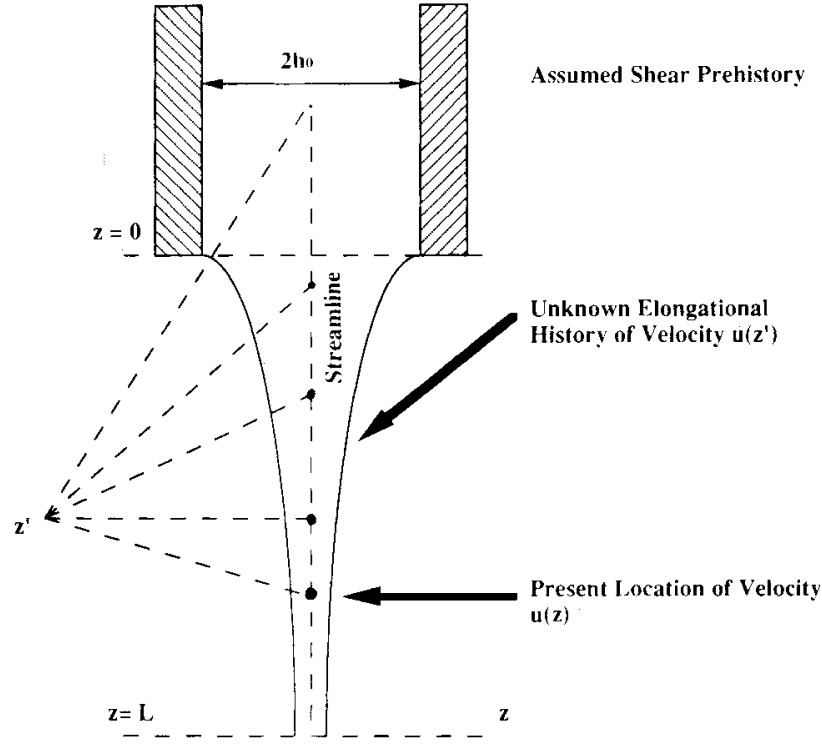

Fig. 2. The present stress depends on the relative deformation to which the fluid particles had been exposed inside and outside the die (prehistory and history, respectively), before reaching its present location, at $z$, i.e., $\tau_{z z}(z)-\tau_{x x}(z)=\int_{-\infty}^{0}() d_{z}^{\prime}+\int_{0}^{z}() d z^{\prime}$.

(b) $B\left[u\left(z^{\prime}\right), u(z)\right]$, between the present location $z$ of velocity $u(z)$ (within the extensional field $0 \leq z \leq l$ ) and past locations in the same extensional field of $0 \leq z^{\prime} \leq z$ of velocity $u\left(z^{\prime}\right)$ :

$$
B\left(u\left(z^{\prime}\right) u(z)\right)=\left[\begin{array}{ccc}
{\left[\frac{u\left(z^{\prime}\right)}{u(z)}\right]^{2}} & 0 & 0 \\
0 & 1 & 0 \\
0 & 0 & {\left[\frac{u(z)}{u\left(z^{\prime}\right)}\right]^{2}}
\end{array}\right] .
$$

A check for consistency, yields $\operatorname{det} B=1$, consistent with incompressibility, and $\mathrm{I}_{B}=\mathrm{II}_{B}$, consistent with the biaxial deformation, for both tensors. In nonisothermal casting, the effects of the temperature history has to be taken into account by the employed constitutive equation. The temperature dependency of a "thermo-rheologically simple" material can be represented by a temperature shift factor, $a_{T}$, which relates the stress relaxation time $\lambda$ at temperature $T$ to that at a reference temperature $T_{0}$. The relaxation time $\lambda$ at timperature $T$ is related to the relaxation time $\lambda_{0}$ at the reference temperature $T_{0}$, by $\lambda=a_{T} \lambda_{0}$. The shift factor $a_{T}$ is obtained from the Arrhenius equation 17.

$$
a_{T}=\exp \left[\frac{E}{R}\left(\frac{1}{T}-\frac{1}{T_{0}}\right)\right]
$$

and is introduced in the constitutive equation through the concept of "pseudo-time" as suggested by Morland and Lee (8), according to which isothermal times, $t$, are rescaled to pseudo-times,

$$
\xi(t)=\int_{0}^{t} a_{T}^{-1} d t^{\prime}
$$


and

$$
d \xi=a_{T}^{-1} d t .
$$

The shift factor and the pseudo-time are introduced into $E q 8$ in place of $t$ and $t^{\prime}$ to account for the nonisothermal strain history as determined by the simultaneously solved energy equation, Eq 4. Thus the non-isothermal form of the constitutive equation takes the form

$$
\begin{aligned}
& \tau=\int_{-\infty}^{z} \sum_{k=1}^{k} \frac{a_{k}}{\lambda_{k}} \exp \left(-\frac{\xi(z)-\xi\left(z^{\prime}\right)}{\lambda_{k}}\right) \\
& \cdot \frac{\alpha}{(\alpha-3)+\beta \mathrm{I}_{B}+(1-\beta) \mathrm{II}_{B}}\left(B_{t}\left(t^{\prime}\right)-\mathrm{I}\right) \frac{d z^{\prime}}{a_{\mathrm{T}} u\left(z^{\prime}\right)},
\end{aligned}
$$

which is also broken into prehistory and history parts along the lines of $E q 14$.

\section{SOLUTION OF EGUATIONS}

The unknown film thickness in the momentum $E q 3$ is eliminated by means of the continuity equation, $E q 1$, which results in

$$
\frac{d}{d z}\left(\frac{\tau_{z z}-\tau_{x x}}{\rho u}\right)=0 .
$$

with $\rho$ given by $E q 2$, and $\tau_{z z}-\tau_{x x}$ by $E q 18$. The stress difference, $\tau_{z x}-\tau_{x x}$, are cross-section averaged, along the lines followed by Matsui and Bogue (18). Equation 21 and the energy equation, $E q 4$, are solved simultaneously by the Galerkin finite element method. The unknown axial velocity $u$ and the temperature $T$ along the film are approximated by a series expansion by means of known quadratic finite-element basis functions, $\phi^{t}$, by

$$
u(z)=\sum_{i=1}^{N} u_{i} \phi^{i}(z),
$$

and

$$
T(z)=\sum_{i=1}^{N} T_{i} \phi^{t}(z) .
$$

respectively, where $2 N$ is the number of the total nodal unknowns, $u_{t}$ and $T_{t}$, to be determined. Then, Eqs 21 and 4 are weighted integrally with the basis functions and the resulting weighted residuals, $R_{i}$, are required to vanish, i.e.,

$$
\begin{aligned}
R_{u}^{\ell}= & \int_{0}^{1} \frac{d}{d z}\left(\frac{\tau_{z z}-\tau_{x x}}{u(z)}\right) \\
& \cdot \phi^{i} d z=0, \quad i=1,2, \ldots, N, \\
R_{T}^{t}= & \int_{0}^{1}\left(\rho c u \frac{d T}{d z}+\frac{H}{h}\left(T-T_{a}\right)\right) \\
& \cdot \phi^{i} d z=0, \quad i=1,2, \ldots, N,
\end{aligned}
$$

where $R_{u}^{i}$ are the momentum and energy residuals, respectively. These equations are simplified further by means of the divergence theorem, to

$$
\begin{aligned}
R_{u}^{i}= & {\left[\frac{\tau_{z z}-\tau_{x x}}{u(z)} \phi^{i}\right]_{z=1} } \\
& -\left[\frac{\tau_{z z}-\tau_{x x}}{u(z)} \phi_{i}\right]_{z=0}-\int_{0}^{1}\left(\frac{\tau_{z z}-\tau_{x x}}{u(z)} \frac{d \phi^{i}}{d z}\right) d z=0,
\end{aligned}
$$

with the stress difference given by Eq 14, and

$$
\begin{aligned}
R_{T}^{i}=\left[T \phi^{i}\right]_{z=1} & -\left[T \phi^{i}\right]_{z=0} \\
& -\int_{0}^{1}\left(\rho c u T \frac{d \phi^{i}}{d z}+\frac{H}{h}\left(T-t_{a i r}\right)\right) d z=0,
\end{aligned}
$$

respectively. The boundary residual at the inlet becomes zero (to specify the velocity at the inlet)

$$
R_{u}^{1}=0,
$$

At the take-up end, either the draw-ratio or the force are known. To specify the draw-ratio which is also the Nth nodal velocity unknown, its residual is set to zero.

$$
R_{u}^{N}=0,
$$

Alternatively, to specify the drawing force the first term of $E q 26$ is replaced by the value of $F$. Similarly, the first boundary energy residual, $R_{T}^{1}$, becomes

$$
R_{T}^{1}=0,
$$

in order to impose the known temperature at the inlet. These weighted residuals required to vanish, results in a system of $2 \mathrm{~N}$ non-linear, algebraic equations. To solve these equations full Newton-iteration was employed

where

$$
q^{(n+1)}=q^{(n)}-J^{-1} R\left(q^{(n)}\right),
$$

$$
q=\left\{u_{1}, T_{1}, u_{2}, T_{2}, \ldots, u_{N}, T_{N}\right\},
$$

is the matrix vector of the unknowns, $R$ is the Galerkin weighted residual vector

$$
R=\left\{R_{t}, R u\right\}^{T},
$$

and $J$ is the Jacobian matrix of the derivatives of the residuals $R$ with respect to the nodal velocities and temperatures,

$$
J=\frac{\partial R}{\partial q} .
$$

\section{RESULTS AND DISCUSSION}

The results are presented in terms of the following dimensionless numbers:

Weissenberg number:

$$
W e=\dot{\epsilon} \frac{\sum a_{k} \lambda_{k}^{2}}{\sum a_{k} \lambda_{k}}=\dot{\epsilon} \bar{\lambda},
$$


Deborah number:

$$
\lambda=\frac{u_{0}}{L} \frac{\sum a_{k} \lambda_{k}^{2}}{\sum a_{k} \lambda_{k}}=\frac{u_{0}}{L} \bar{\lambda},
$$

Drawing force:

$$
f=\frac{F L}{\mu Q},
$$

Draw ratio:

$$
\operatorname{Dr}=\frac{u(z=1)}{u(z=0)},
$$

Here, $\dot{\epsilon}=\left(\partial_{u} / \partial_{z}\right)$, is the local extension rate; $\mu=\sum a_{k} \lambda_{k}$ is the zero shear viscosity; and $\bar{\lambda}=\sum a_{k} \lambda_{k}^{2} / \sum a_{k} \lambda_{k}$ is an average relaxation time. The rest of the variables in Eqs 35 to 38 are shown in Figs. 1 and 2. Figure 3 shows that the analytic Newtonian velocity profile is predicted well in the limit of $\lambda=0.01$, as it should, and that solutions at higher elasticities are meshindependent. Figure 4 shows predicted velocity and corresponding film thickness along the film, for liquids of different elasticities and fixed shear-thinning $(\alpha=10)$ at fixed draw ratio $(D r=5)$. The resulting viscoelastic films thin abruptly just downstream the exit-die compared to the Newtonian one under the same conditions due to the normal stress difference that grows with relaxation time. Figure 5 shows the same kind of predictions at fixed relaxation time $(\lambda=4.92)$ and fixed draw ratio $(D r=5)$ for liquids of different shear-thinning degrees. The most shear-thinning liquid $(\alpha=10)$ thins the easiest as compared to the liquid of almost constant viscosity ( $\alpha=1000)$, as expected.

Figure 6 shows calculated viscoelastic stresses at

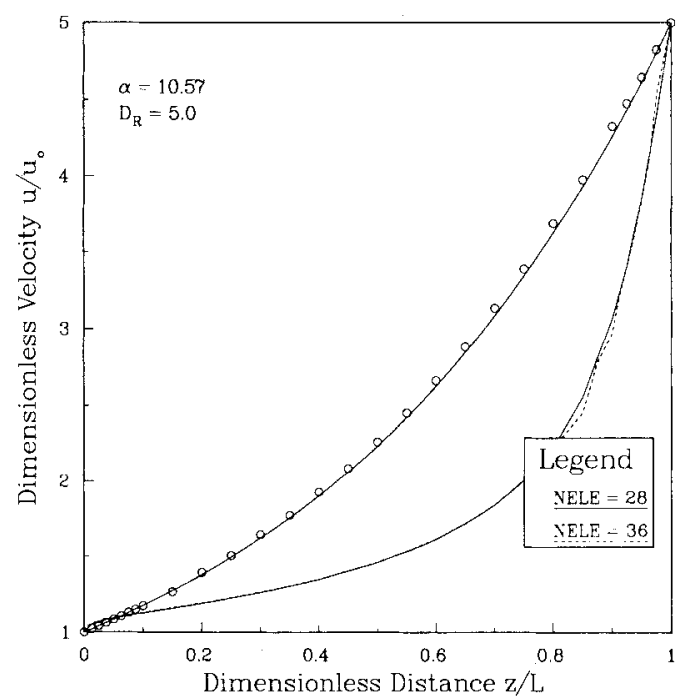

Fig. 3. Finite-element predictions with the integral constitutive equation in the limiting case of, $\lambda=0.01$, agrees with the exact solution for Newtonian liquid, as it should. Also shown is a solution at the same draw ratio of a viscoelastic liquid of relaxation time $\lambda=4.92$ and shearthinning $\alpha=1000$ obtained with two different numbers of elements, which demonstrates a mesh-independent solution.

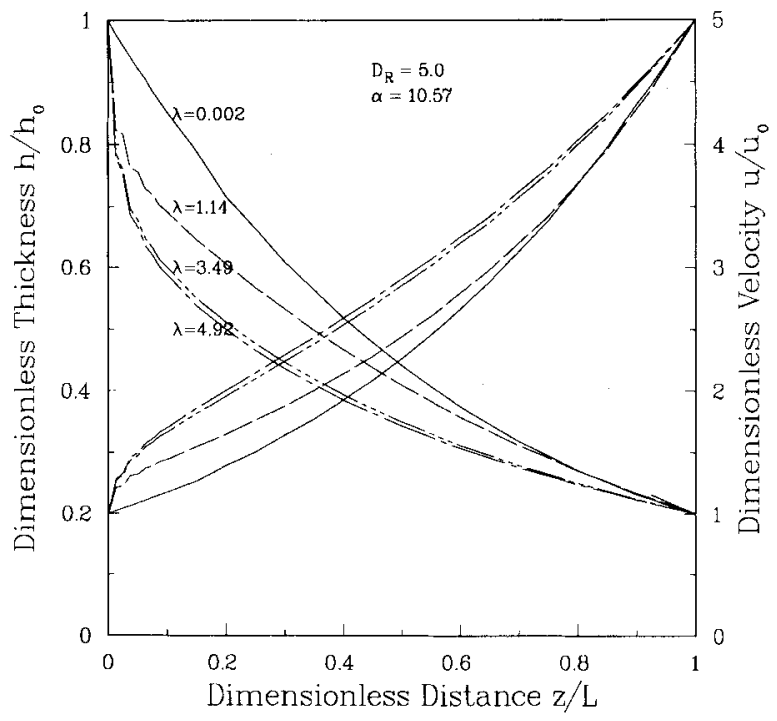

Fig. 4. Finite-element predictions of film and velocity profiles with $\alpha=10.6$ in the constitutive equation (shearthinning (iquid), draw ratio $D_{R}=5$, and several degrees of elastictty represented by the relaxation time $\lambda$, according to $\mathrm{Eq} 35$.

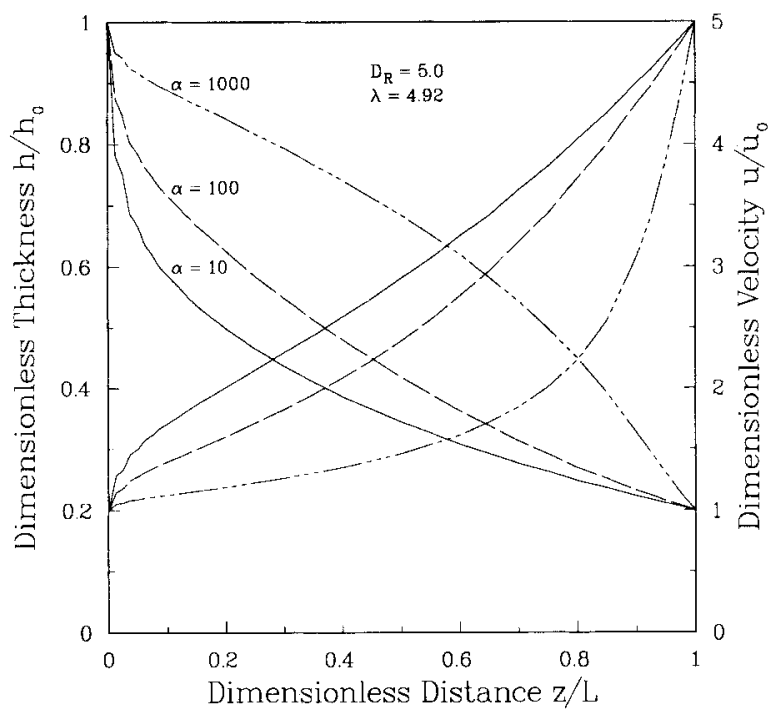

Fig. 5. Predicted velocity and film thickness profiles for different shear thinning materials (with decreasing $\alpha$ ) at fixed relaxation time $\lambda=4.92$, and fixed draw ratio $D_{R}=5$.

the die-exit, at two different locations downstream, and at the take-up end. The axial stress, initially, goes through a maximum (which, depending on the location of the film, occurs at rather low elasticities), and then it decreases as relaxation time increases and finally it diminishes to zero. Similarly, the radial stress also decreases with relaxation time, but unlike the axial one, it decreases monotonically to some finite values. At the die-exit, where the stresses are entirely due to the shear prehistory within the die, the ratio of the two decays from the Newtonian value of one to nearly zero as relaxation time increases. Thus a zero value of this ratio is a good approximation 
to the inlet stress boundary condition required with differential models only at high relaxation time. Figure 7 shows how the predicted required tensions to achieve certain draw ratio, $D r=5$, and therefore certain film thickness, increases with the relaxation time for liquids of several degrees of shear-thinning, which decreases with increasing parameter $\alpha$. All curves depart from the appropriate Newtonian value of $\lambda=0$ independently of $\alpha$. For the Maxwell liquid of constant viscosity $(\alpha=10000)$, the required ten-
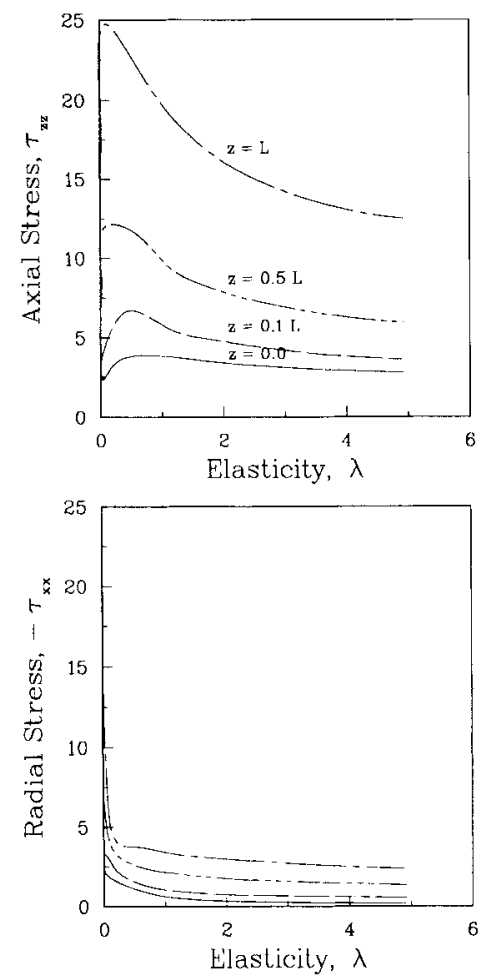

Fig. 6. Development and decay of viscoelastic stresses along the film (for $D_{R}=5$ and $\alpha=100$ ).

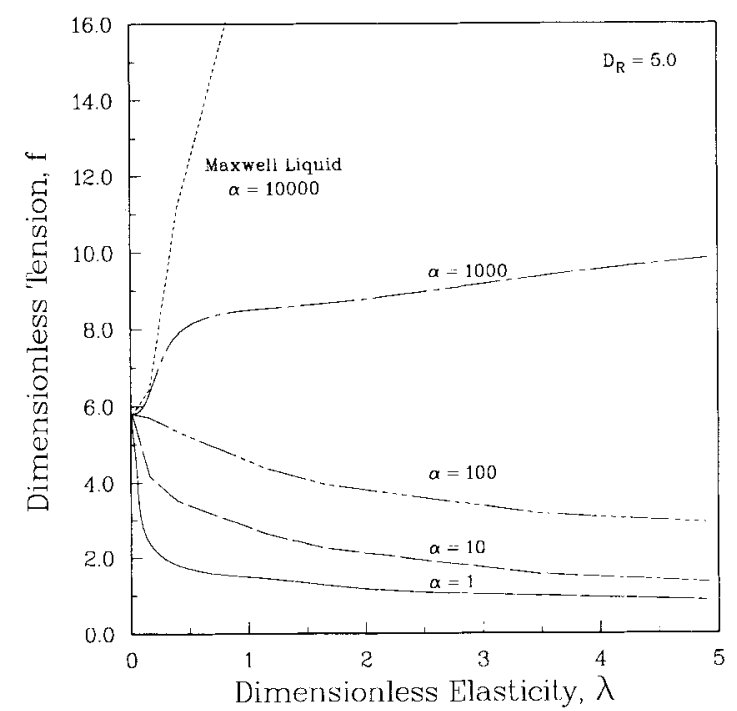

Fig. 7. Predicted tension at fixed draw ratio for viscoelastic liquids of several shear-thinning degrees, denoted by increasing $\alpha$. sion increases with relaxation time and becomes unbounded at $\lambda=1$. The shear-thinning character dominates in the rest of the cases $(\alpha<10000)$ and for highly shear-thinning liquids $(\alpha<100)$ the required tension eventually decreases with relaxation time. Figure 8 shows the predicted required tension to achieve target film thicknesses, at fixed relaxation time $(\lambda=1.14)$ and different shear-thinning degrees. This tension of course increases with decreasing film thickness, and with decreasing shear-thinning. The non-shear-thinning Maxwell liquid requires the largest tensions.

To proceed to non-isothermal casting, the shift factors of the time-temperature superposition is required. Figure 9 shows shift factor of polystyrene

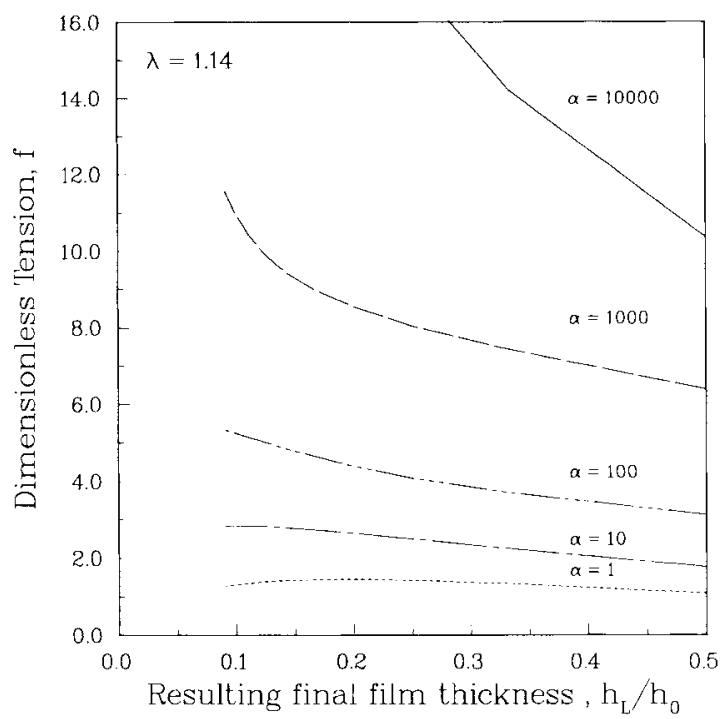

Fig. 8. Predicted tension to cast films to several thickness of viscoelastic liquids of fixed relaxation time $(\lambda=$ 1.14) and several degrees of shear-thinning (increasing with decreasing $\alpha$ ).

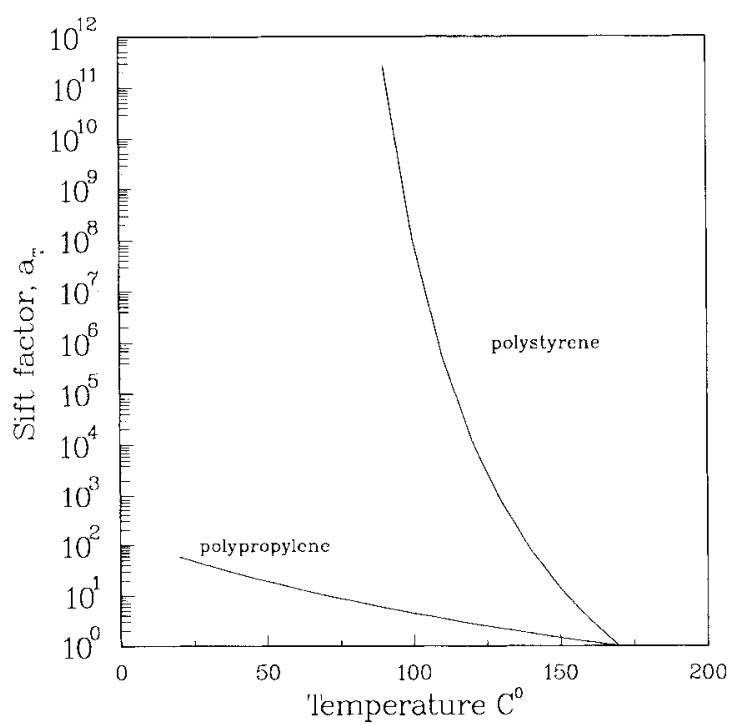

Fig. 9. Shift factors for polystyrene and polypropylene computed from data taken from (21) and (22), respectively. 
and polypropylene produced from data in Refs. 20 and 21 , respectively. Rheological data for polystyrene, required in the constitutive equation were produced from data given in (21), and are shown in Table 2. It should be mentioned that in this analysis any effects contributed to crystallization is neglected. Figure 10 shows predictions corresponding to the characterized polypropylene of properties shown in Table 2 and Fig. 9, at draw ratio $\mathrm{Dr}=10$ and dietemperature, $T=170^{\circ} \mathrm{C}$. Castability is shown to greatly enhance by cooling air. As shown by the corresponding Fig. 11, for the lowest of the air temperature, $T_{\text {atr }}=30^{\circ} \mathrm{C}$, the film solidifies approximately halfway downstream the die-exit (where its thickness does not diminish any more).

Computed temperature profiles, corresponding to Figs. 10 and 11 , are shown in Fig. 12. By comparing Figs. 11 and 12, solidifications indeed occurs at temperature $T_{s}=132$ which is close to the glass transition temperature of the same polystyrene.

\section{COMPARISON WITH EXPERIMENTS}

There are only limited amount of data on film casting in the literature to compare and to evaluate the predictions of this analysis. The most complete

Table 2. Shear Material Parameter and Relaxation Spectrum of Polystyrene at $170^{\circ} \mathrm{C}$, Calculated from (20).

\begin{tabular}{cc}
\hline $\mathbf{a}_{k}$ & \multicolumn{1}{c}{$\boldsymbol{s}$} \\
\hline $\mathbf{P a}$ & $1.77 E+2$ \\
$9.78 E-4$ & $2.09 E+1$ \\
$3.46 E-2$ & $3.93 E 0$ \\
$6.78 E-2$ & $4.39 E-1$ \\
$1.01 E-1$ & $5.05 E-2$ \\
$1.26 E-1$ & $7.55 E-3$ \\
$1.45 E-1$ & $4.55 E-4$ \\
$1.47 E-1$ & 10.57 \\
$\alpha$ &
\end{tabular}

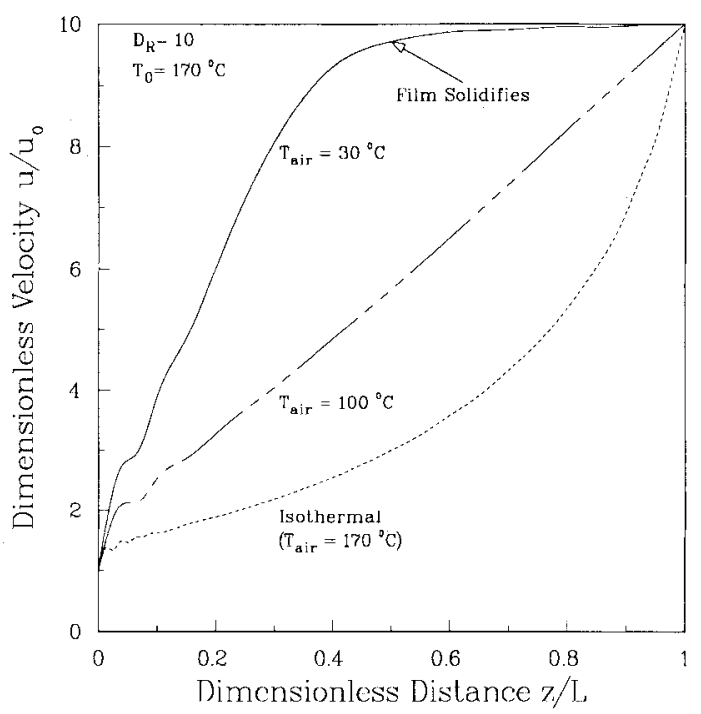

Fig. 10. Predicted velocity profiles for polystyrene of properties shown in Table 2 and Fig. 9, under isothermal and non-isothermal conditions.

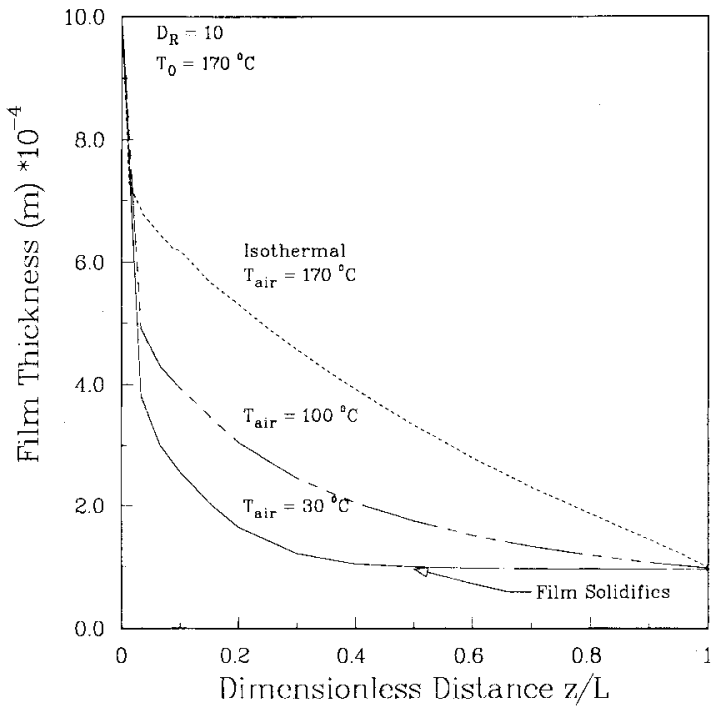

Fig. 11. Predicted film profiles for polystyrene of properties shown in Table 2 and Fig. 9, under isothermal and non-isothermal conditions.

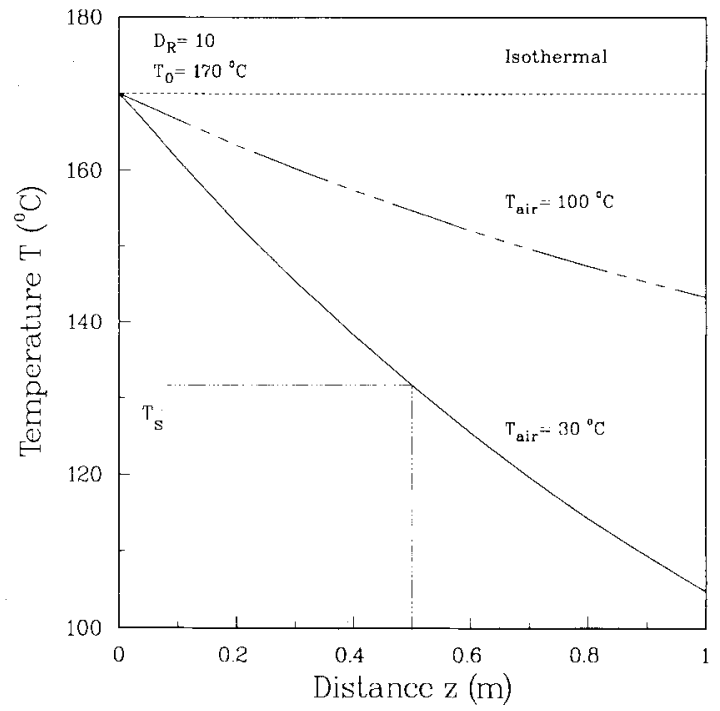

Fig. 12. Predicted temperature profiles for polystyrene of properties shown in Table 2 and Fig. 9.

set of data was given by Kase (3) for casting of polypropylene. The polymer film was cooled by the ambient and was taken up at the chill roll under tension. The conditions of the process are shown in Table 3. Ishizuka, et al. (21), in a comprehensive work on fiber spinning of polypropylene, presented complete rheological data for the specific polypropylene, from which the relaxation spectrum is calculated, as shown in Table 4. Then, fitting the experimental data of shear viscosity $v$ s. shear rate, also given by Ishizuka, et al. (21), the shear-thinning material parameter, $\alpha$, of the constitutive equation was found to be 10. As mentioned earlier, the first and second invariants of the Finger tensor for biaxial extension under casting conditions are equal and therefore the 
second material parameter, $\beta$, of the constitutive equation is not needed. The shift factor, $a_{T}$, for polypropylene was calculated by the Arrhenius equation and is plotted in Fig. 9. Figure 13 shows the predictions of this analysis compared with the experimental data of Kase (3) for two different takeup velocities, $0.35 \mathrm{~m} / \mathrm{s}$ and $0.67 \mathrm{~m} / \mathrm{s}$, and other conditions as shown in Table 3 . Apart from the two ends of the film, at the die-exit and at the take-up, where the one-dimensional analysis may fail due to flow rearrangements and apparent contact with the chill roll, respectively, the predictions agree with the recorded thickness profiles. The corresponding predicted and measured temperature profiles are shown in Fig. 14. This prediction for temperature profile was obtained when the coefficients were $a=0.2$ and

Table 3. Film Casting Condition for Polypropylene Taken from (3).

\begin{tabular}{lc}
\hline Effective Die Opening & $1000 \mu$ \\
Inlet Temperature & $215^{\circ} \mathrm{C}$ \\
Air Temperature & $30^{\circ} \mathrm{C}$ \\
Film Thickness & $30 \mu$ \\
Take-up Speed & $0.35 \mathrm{~m} / \mathrm{s}$ \\
Draw-down Ratio & 33 \\
\hline
\end{tabular}

Table 4. Relaxation Spectrum of Polypropylene at $215^{\circ} \mathrm{C}$ Calculated from (21).

\begin{tabular}{cc}
\hline$a_{k}$ & $\lambda$ \\
$\mathbf{P a}$ & $\mathbf{s}$ \\
\hline $8.27 E+0$ & 0.1 \\
$8.22 E-1$ & 1 \\
$1.10 E-2$ & 10 \\
$2.33 E-6$ & 100 \\
$3.19 E-12$ & 1000 \\
\hline
\end{tabular}

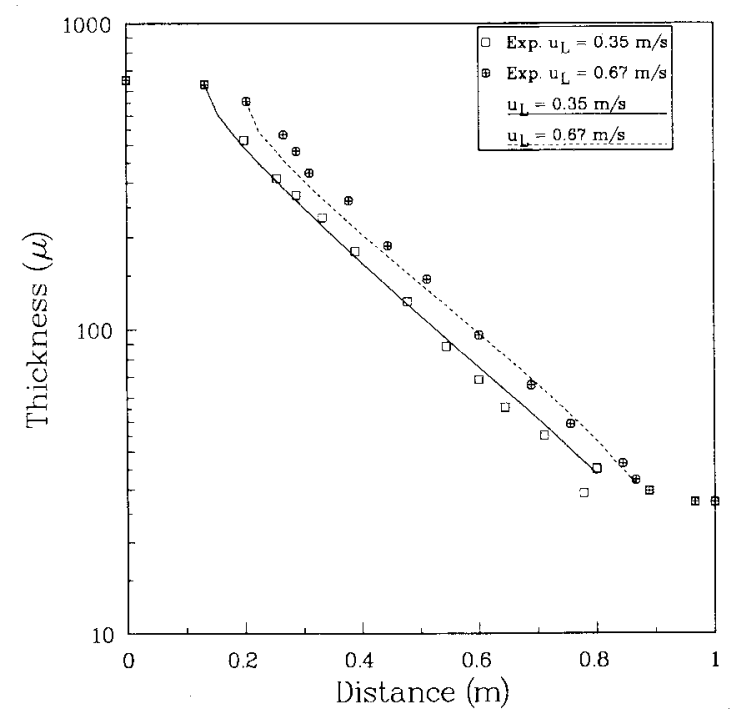

Fig. 13. Comparison between predicted film thickness and experimental data taken from Kase (3), under two different take-up velocitles, and conditions shown in Table 3, of polypropylene of properties shown in Table 4. $b=0.6$ in Eqs 6 and 7, respectively. It has been suggested that the process could be analyzed by neglecting viscoelasticity and considering only a temperature-dependent elongational viscosity. In order to evaluate the accuracy of this approximation, we used an Arrhenius type equation, to present the dependency of viscosity on temperature, with activation energy of $5100 \mathrm{~K}$ from (22). Figure 15 shows the results of such an approximation. Obviously, viscoelasticity has a significant effect on the behavior of the fluid and can not be neglected.

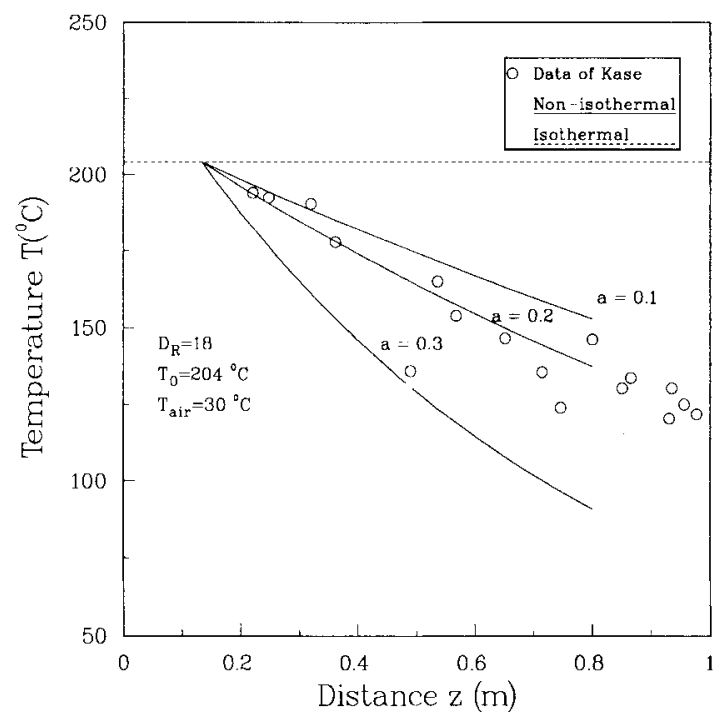

Fig. 14. Comparison between predicted temperature profile and experimental data from Kase (3). for polypropylene of properties shown in Table 4 and casting conditions shown in Table 3, where $a$ is the coefficient in Eqn. (6).

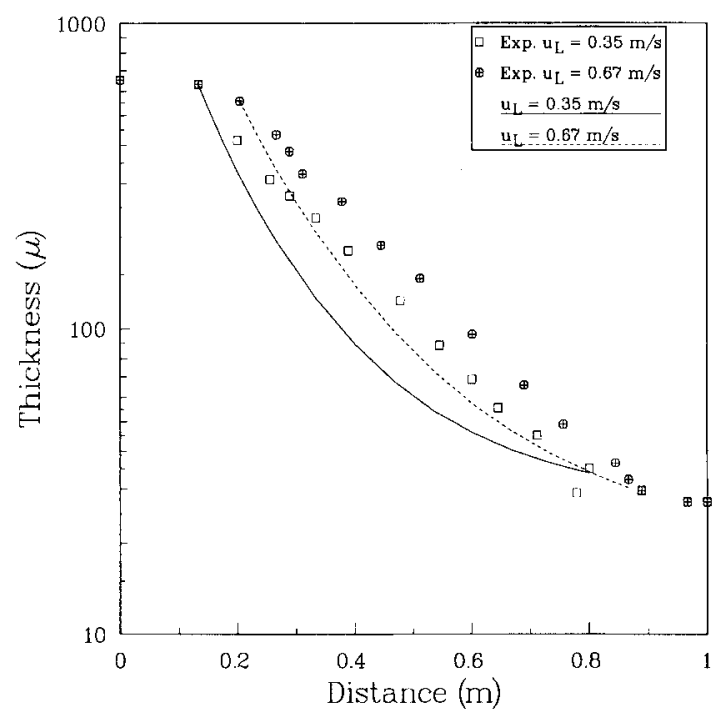

Fig. 15. Comparison between predicted film thickness, by means of a temperature-dependent viscosity and neglecting viscoelasticity, and experimental data taken from Kase (3), under two different take-up velocities, and conditions shown in Table 3, of polypropylene of properties shown in Table 4. 


\section{CONCLUSION}

Isothermal and non-isothermal film casting were investigated by means of a nonlinear, integral constitutive equation of BKZ-type, finite elements, and Newton iteration. Thinning of the extruded film is enhanced by shear-thinning rheology and by cooling air often down to solidification at about the glass transition temperature, upstream the chilling roll. The required tension decreases with shear-thinning and increases with relaxation time. The temperature profile along the film departs from linear as the temperature of the cooling air decreases to about the ambient. Predicted thickness and temperature profiles agree with experimental data of casting of polypropylene taken from the literature.

\section{NOMENCLATURE}

$a=$ Coefficient in heat transfer correlation.

$a_{k} \quad=$ Relaxation coefficient, $P a$.

$a_{T} \quad=$ Shift factor.

$c_{\rho} \quad=$ Constant of expansion.

$c_{p}=$ Specific heat capacity, $\mathrm{J} \mathrm{kg}^{-1 . \mathrm{K}-1}$.

$c_{p}^{0}=$ Specific heat capacity at a reference temperature, $\mathrm{J}^{\mathrm{kg}} \mathrm{g}^{-1 . \mathrm{K}-1}$.

Dr $=$ Draw ratio.

$g=$ Gravity acceleration, $\mathrm{m} \cdot \mathrm{s}^{-2}$.

$h \quad=$ Film Thickness, $\mathrm{m}$.

$h_{0}=$ Die-half thickness, $\mathrm{m}$.

$H \quad=$ Heat transfer coefficient, J.m ${ }^{-2 . \kappa-1}$.

$J \quad=$ Jacobian matrix.

$k_{1}, k_{2}=$ Empirical constants.

$K=$ Thermal conductivity, J. $\mathrm{m}^{-1 \cdot K-1}$.

$L \quad=$ Length of the film, $m$.

$m \quad=$ Power law coefficient.

$n \quad=$ Power law exponent.

$p=$ Pressure, $\mathrm{Pa}$.

$q \quad=$ Matrix vector of unknowns.

Q $=$ Volumetric flow rate, $\mathrm{m}^{3 . \mathrm{s}-1}$.

$T=$ Film temperature, $\mathrm{K}$.

$t \quad=$ Traveling time, $\mathrm{s}$.

$u=$ Film velocity, $\mathrm{m} \cdot \mathrm{s}^{-1}$.

$v \quad=$ Film velocity inside the die, m.s.

$w \quad=$ Film width, $\mathrm{m}$.

$=$ Radial direction.

$=$ Flow direction.

\section{Greek Letters}

$\alpha=$ Shear material parameter.

$\beta=$ Extension material parameter.

$\epsilon=$ Extension rate, $\mathrm{s}^{-1}$.

$\xi \quad=$ Scaled time, $\mathrm{s}$.

$\rho=$ Density, $\mathrm{kg} \cdot \mathrm{m}^{-3}$.
I, $\Pi=$ First and second invariants of the Finger tensor.

$\lambda=$ Relaxation time, $\mathrm{s}$.

$\phi=$ Basis function.

\section{Superscripts}

$i=i$ th basis or unknown.

$\mathrm{n}=$ Iteration number.

$T=$ Transpose.

\section{Subscripts}

$a=$ Properties of air.

$k=$ Number of relaxation times.

$u=$ Residuals of momentum equation.

$T=$ Residuals of energy equation.

\section{REFERENCES}

1. J. R. A. Pearson, Mechanics of Polymer Processing. Elsevier Applied Science Publishers, London and New York (1985).

2. N. S. Clarke. Mathematika, 12, 51 (1968).

3. S. Kase, J. Appl. Polym. Sci., 18, 3279 (1974).

4. N. R. Anturkar and A. Co, J. Non-Newt. Fluid Mech., 28, 287 (1988).

5. S. F. Kistler and L. E. Scriven, "Coating Films," in Computational Analysts of Polymer Processing, J. R. A. Pearson and S. M. Richardson, eds., Applied Science Publishers Ltd., London and New York (1983).

6. K. Adachi, T. Aoki, S. Nishida, and R. Nakamura, "A Hydrodynamic Investigation of Falling Liquid Film for Curtain Coating and Sheet Casting," Xth International Congress on Rheology, Sydney (1988).

7. T. C. Papanastasiou, L. E. Scriven, and C. W. Macosko, J. Rheol., 27, 387 (1983).

8. L. W. Morland and E. H. Lee, Trans. Soc. Rheol., 4, 233 (1960).

9. B. Bernstein, E. K. Kearsley, and L. J. Zapos, Trans. Soc. Rheol., 7, 391 (1963).

10. M. H. Wagner, Rheol. Acta., 18, 33 (1979).

11. M. Dol and S. F. Edwards, J. Chem. Soc. Farady Trans., 74, 918 (1979).

12. C. F. Curtiss and R. B. Bird, J. Chem. Phys., 74, 2026 (1981).

13. C. J. S. Petrie, Elongational Flows, Pitman, London (1979).

14. J. Haw, PhD thesis, Polytech. Inst. of New York (1984).

15. R. H. Perry and C. H. Chilton, Chemical Engineer's Handbook, McGraw-Hill, New York (1973).

16. S. Kase and T. Matsuo, J. Appl. Polym. Sci., 11, 251 (1967).

17. J. Meissner, in G. Schreyer, Konstruieren mit Kunststoffen, Munich (1972).

18. M. Matsui and D. C. Bogue, Polym. Eng. Sci., 16, 735 (1976).

19. Z. Chen and A. C. Papanastasiou. Intern. Polym. Proc., 2, 33 (1987).

20. J. D. Ferry, Viscoelastic Properties of Polymers, Wiley \& Son, London (1961).

21. O. Ishizuka and K. Koyama, Polymer, 21, 671 (1980).

22. R. I. Tanner, Engineering Rheology, Clarendon Press, Oxford (1985). 